\title{
"Polluit sanguis iste, non redemit" - Beobachtungen zum Motiv des Blutes in Firmicus Maternus, De errore profanarum religionum
}

Bettina Reese

(Martin-Luther-Universität Halle-Wittenberg)

\begin{abstract}
"Polluit sanguis iste, non redemit" - Observations concerning the motif of blood in Firmicus Maternus, De errore profanarum religionum
\end{abstract}

\begin{abstract}
In his De errore profanarum religionum, Firmicus Maternus fiercely attacks the traditional cults of the Roman Empire as well as the mysteries, which he considers to be the invention of the devil and a complete perversion of Christian sacraments. This paper analyses the motif of blood in his work. A close reading of the text reveals that Firmicus applies dichotomic functions to the use of blood in ritual contexts, such as poison and remedy, purity and pollution, and salvation and damnation. These dichotomies serve two purposes for him: First, they allow him to construct clear boundaries between the pagan religion (deliberately constructed as 'one' system and suppressing its diversity) and Christianity as two opposing religious systems. Second, by turning the tables, they allow him to undermine the traditional conceptions concerning ritually used blood. Thus, he presents 'pagan' blood as being venomous, polluting, and only leading on the road to perdition, whereas only the blood of Christ truly has the power to heal, to cleanse, to redeem, and to grant eternal salvation.
\end{abstract}

\section{Keywords}

Firmicus Maternus; De errore profanarum religionum; blood, ritual use of; mysteries; purity and pollution; salvation

Der Aufsatz geht auf einen Vortrag im Rahmen der Laetae segetes VII in Brno zurück. Den Veranstaltern der Tagung sei an dieser Stelle nochmals herzlich gedankt. Als Textgrundlage der Mathesis werden die Ausgaben von Kroll et al. (1968ab) und Monat (1994; 2002; 2003) herangezogen, für De errore die Ausgaben von Ziegler (1953a) und Turcan (2002). 


\section{Einleitung}

Die Schrift De errore profanarum religionum des Iulius Firmicus Maternus hebt sich von anderen apologetischen Schriften ${ }^{1}$ vor allem durch ihren aggressiven Tonfall und den unverhohlenen Aufruf zur Vernichtung der nichtchristlichen Kulte ab, was zu kontroversen und häufig recht negativen Bewertungen des Werks wie auch des Autors geführt hat. ${ }^{2}$ Aufgrund des Umfangs, in dem sich Firmicus mit den Mysterienkulten auseinandersetzt, wurde die Schrift zudem als Quelle für verschiedene Mysterien, wie z.B. jene der Kybele und des Attis, herangezogen. Neuere Studien widmen sich beispielsweise Firmicus' religiösen Überzeugungen vor seiner Konversion und insbesondere seinem Verhältnis zu den ,Anderen', die in De errore als Gegner ausgemacht werden. ${ }^{3}$

Ein Aspekt, der meines Erachtens bisher noch nicht ausreichend berücksichtigt wurde, ist die Verwendung des Motivs Blut, das in seinem Werk zwei Funktionen erfüllt: Einerseits erlaubt es der Einsatz dieses Motivs dem Autor, die Opposition zwischen Christentum und ,Heidentum‘ als zweier konkurrierender religiöser Systeme deutlicher hervortreten zu lassen. ${ }^{4}$ Andererseits unterläuft er mit seiner Darstellung die Vorstellungen, die an die Wirkung des Blutes in den traditionellen Kulten geknüpft waren und verkehrt sie ins Gegenteil, um diese insgesamt als Ansammlung unheiliger, verunreinigender und letztendlich verderbenbringender Irrtümer darstellen zu können.

\section{Firmicus Maternus: Leben und Werk}

Firmicus' Leben lässt sich nur in groben Zügen anhand der wenigen autobiographischen Hinweise aus der Mathesis und De errore profanarum religionum rekonstruieren. Er dürfte um 300/10 auf Sizilien, möglicherweise in Syrakus, geboren worden sein und entstammte einer wohlhabenden Familie. ${ }^{5}$ Er war zunächst als Anwalt tätig, zog sich aber aus diesem Beruf zurück, nachdem er sich eigenen Angaben nach wiederholt den An-

1 Ob und inwiefern Firmicus' Schrift als Apologie bezeichnet werden kann, kann an dieser Stelle nicht diskutiert werden. De errore wird mehrheitlich den Apologien zugeordnet, auch wenn innerhalb der Schrift das Christentum nicht mehr im eigentlichen Sinne verteidigt wird und der Fokus gänzlich auf dem Angriff auf die traditionellen Kulte liegt; cf. z.B. Ahmed (2017: p. 168) oder Hübner \& Wlosok (1989: p. 92).

2 Firm. err. 16,4f.; 20,7; 28,6 und 29,1. Die Neuheit dieses Appells betonen Hübner \& Wlosok (1989: pp. 89-92). Festugière, der den Firmicus der Mathesis als „dévot païen“ einschätzt, bedauert dessen Haltung in err. und beschreibt ihn als „chrétien résolu, imbu d'une ardeur farouche et d'un esprit de revanche“ (1998: p. 9); Turcan hält Firmicus' „militantisme forcené“ für eine Strategie, um seine Vergangenheit als Astrologe in Vergessenheit geraten zu lassen (2002: p. 30) und Gaddis attestiert De errore extremistische Züge (2005: p. 164). Einen Überblick über die differierenden Einschätzungen des Autors bieten Caseau (2007: pp. 43-46) und Gassman (2020: p. 58 mit Anm. 72-73).

3 Zur religiösen Überzeugung vor seiner Konversion cf. z.B. Caseau (2007); zum Verhältnis zu den Anderen cf. z.B. Massa (2013) und Ahmed (2017: pp. 164-200).

4 Massa (2013: p. 507) und im Anschluss daran Gassman (2020: p. 60).

5 So z.B. Monat (2002: p. 8) oder Turcan (2002: p. 12). Zur Herkunft von Sizilien: Firm. math. I, pr. 4 und aus Syracus: math. VI,30,26, der Lesart „civis meus“ (Kroll et al. 1968: p. 148) folgend. Für die Zugehörigkeit zu einer angesehenen Familie spricht u. A. die Freundschaft zu Lollianus signo Mavortius, praef. urbis 
feindungen anderer Personen ausgesetzt sah (Firm. math. IV, pr. 1-3). Das Forum und irdische Angelegenheiten hinter sich lassend, wandte er sich Höherem zu und verfasste zwischen 334-337 die acht Bücher umfassende, astrologische Schrift Mathesis. ${ }^{6}$ Nach seiner Konversion zum Christentum veröffentlichte er zwischen 343 und 350 das apologetische Werk De errore profanarum religionum. ${ }^{7}$

Obwohl der Anfang der Schrift De errore verloren ist, lassen sich die Kaiser Constans und Constantius als primäre Adressaten der Schrift bestimmen, die über den ganzen Text hinweg wiederholt als sacratissimi imperatores angesprochen werden. ${ }^{8}$ Als eine weitere Gruppe, die sich möglicherweise von der Schrift angesprochen fühlte, zieht Ahmed Personen in Betracht, die bereits am Sinn der traditionellen Kulte zweifelten. ${ }^{9}$ Dabei dürfte es sich wohl am ehesten um gebildete Anhänger der Oberschicht gehandelt haben, die u. A. mit der Mythenallegorese vertraut waren. Überzeugte Anhänger der traditionellen Kulte hingegen dürfte De errore wohl kaum erreicht haben, nicht nur wegen des den Nichtchristen gegenüber angeschlagenen Tonfalls, sondern auch, weil Firmicus die christliche Position mit Hilfe der Bibel begründet, die von nichtchristlicher Seite nicht als zentrale Autorität akzeptiert wurde. ${ }^{10}$

In den ersten Kapiteln (2-5) von De errore profanarum religionum setzt sich Firmicus zunächst mit der Vergöttlichung der Elemente auseinander (Kapitel 2-5), bevor er eine Reihe von Kulten, darunter den des Liber und der Libera oder den der Persephone attackiert, die von ihm in euhemeristischer Lesart als Totenkulte (funera) gedeutet werden (Kapitel 6-17). Im Anschluss daran wendet er sich den Mysterienkulten und deren symbola zu, die er allesamt für teuflische Nachahmungen der christlichen Sakramente hält (Kapitel 18-27). ${ }^{11}$ Der Text schließt mit dem Aufruf an die Kaiser, die traditionellen Kulte zu verfolgen und auszurotten, da diese sich des Verbrechens des Götzendienstes

342 und comes ordinis primi, dem Widmungsempfänger der Mathesis (PLRE 1, 1987: p. 513, s.v. Lollianus 5 und math. I pr. 1). Einige Handschriften führen Firmicus darüber hinaus als vir clarissimus.

6 Firm. math. IV, pr. 3: caelestibus me ac divinis disputationibus applicarem. Die Datierung der Schrift ergibt sich aus der in math. I,4,10 erwähnten Sonnenfinsternis des Jahres 334 und der Tatsache, dass Firmicus in I,10,13 von Constantin als noch lebendem Kaiser spricht.

7 Firmicus spricht in err. 20,7 Constans und Constantius namentlich an. Zudem spielt er in err. 28,6 auf die Britannienexpedition des Constans 343 an (et insperatam imperatoris faciem Britannus expavit), woraus sich ein Abfassungszeitraum zwischen 343 und 350 ergibt. Eine weitere Eingrenzung auf 346-350 ziehen u. A. Pastorino (1969: ad loc.) und Turcan (2002: ad loc.) in Betracht, die in der Formulierung Persica vota conlapsa sunt (err. 29,3) eine Anspielung auf die Aufhebung der Belagerung von Nisibis 346 sehen; eine zurückhaltendere Position diesbezüglich dagegen bei Ziegler (1953a: p. 6).

8 Der Text beruht auf einer einzigen Handschrift, dem Codex Palatinus Latinus 165, dessen erste zwei Folia ausgefallen sind, cf. Ziegler (1953a: p. 20). Die Anreden als sacratissimi imperatores finden sich in err. 6,1; 7,$7 ; 8,4 ; 16,3 ; 25,4$ und 29,1; in 20,7 werden beide Kaiser namentlich angesprochen. Auf die panegyrischen Züge der wiederholten Kaiseranreden hat Woudhuysen hingewiesen. Seiner Interpretation nach bezieht sich Firmicus vor allem auf Constans, den Augustus des Westens (Woudhuysen 2018: pp. 172, 174).

9 Ahmed (2017: pp. 178f.). Sie verweist auf eine Passage in err. 8,5, in der zwischen unbelehrbaren Anhängern der traditionellen Kulte und denen unterschieden wird, die zwar errantes oder dubitantes seien, nichtsdestotrotz aber noch gerettet werden könnten.

10 Schon Laktanz hatte in Div. Inst. V,4,4 die Überzeugungskraft der Bibel für Nichtchristen in Frage gestellt.

11 Firm. err. 18,1 und 22,1: perversa diaboli ... imitatione. 
(idolatriae facinus, err. 29,1) schuldig gemacht hätten. Firmicus ist damit der erste christliche Autor, der gewaltsames staatliches Vorgehen zur Unterdrückung und Vernichtung der traditionellen Kulte für legitim hält. ${ }^{12}$

Trotz des schärferen Tonfalls in De errore haben jüngere Forschungsbeiträge zu Recht darauf hingewiesen, dass die in der Mathesis und in De errore vertretenen Positionen nicht so weit auseinanderliegen, wie es auf den ersten Blick scheint. ${ }^{13}$ Neben der Beschreibung des höchsten Gottes (summus deus) sei an dieser Stelle auf das Thema Reinheit hingewiesen, das in beiden Werken eine wichtige Rolle einnimmt. ${ }^{14}$ So gibt Firmicus an, die Bücher der Mathesis mit reinem Geist und frei jeglicher Befleckung verfasst zu haben und fordert Lollianus dazu auf, sich ebenfalls von irdischer Unreinheit frei zu halten, um so das Göttliche der Seele Gott wieder nahe bringen zu können. ${ }^{15}$

\section{Die Gegner in De errore profanarum religionum}

Firmicus' Kritik der nichtchristlichen Kulte trifft, jeweils in euhemeristischer Deutung, mit den offiziellen Kulten und den Mysterien sacra publica und sacra privata gleichermaBen. Und auch wenn die Mysterien bereits bei anderen Apologeten ein Ziel der Kritik gewesen waren, stellt ihre systematische, vergleichende Behandlung ein Alleinstellungsmerkmal der Schrift dar, denn Firmicus erhebt den Anspruch, nichts weniger als alle Symbole der Mysterien (omnia symbola profanae religionis, err. 21,1) betrachten und als teuflische Erfindungen entlarven zu wollen. ${ }^{16}$ Während die Mysterien, auf die er sich bezieht, an einigen Passagen klar identifiziert werden können, bleibt an anderen Stellen hingegen offen, auf welche Kulte seine Kritik abzielt. Forscher haben daher die Struktur und Kohärenz der Schrift bemängelt und die Überzeugungskraft der Argumentation, die auf der Gegenüberstellung der nichtchristlichen symbola mit Passagen aus der Heiligen Schrift fußt, in Frage gestellt. ${ }^{17}$ Es stellt sich jedoch die Frage, ob Firmicus' Fokus tatsächlich auf den einzelnen Mysterien en détail liegt oder ob seine Darstellung nicht vielmehr andere Ziele verfolgt.

12 Firm. err. 16,4f.; 20,7; 28,6 und 29,1.

13 So z.B. Ahmed (2017: pp. 165f.) und Gersh (2018: p. 1634).

14 Die Bezeichnung summus deus tritt beispielsweise in math. I,5,7 und II,30,5 sowie in err. 2,4; 4,4 und 29,4 auf. In math. $\mathrm{V}$ praef. 3 wird der höchste Gott als solus omnium gubernator et princeps, solus imperator ac dominus angesprochen. Zur Reinheit in De err. cf. unten, ab Anm. 32ff.

15 Firm. math. V praef. 4: pura mente et ab omni terrena conuersatione se posita et cunctorum flagitiorum labe purgata. Für die Aufforderung zur Reinheit, vgl. z.B. Firm. math. II,30,15 und insbesondere VIII,1,1: Nihil aliud in hac uitae breuitate laborandum nobis est, Mauorti decus nostrum, nisi ut, terreni corporis labe purgata et amputatis si fieri potest omnibus uitiis, uel certe plurimis, incorruptam animi diuinitatem et nulla scelerum contagione pollutam auctori nostro reddamus deo, ne diuinae fabricationis immemorem animum nostrum, uitiosis libidinum laqueis implicatum, tamquam proiectum per praecipitia perdamus.

16 Massa (2013: pp. 502, 504). Die Mysterien erwähnen u. A. auch Justin, 1 apol. 66,4 und Tert. praescr. 40,2-4.

17 Turcan (2002: pp. 34f.) und Forbes (1970: p. 20). 


\section{Das Motiv Blut in De errore profanarum religionum}

Firmicus verwendet verschiedene Bilder, um die traditionellen Kulte zu charakterisieren.

Neben der Bezeichnung der Götterverehrung als Totenkult (funera) und der Tempel als Grabstätten (busta) verwendet er auch das Adjektiv funestus, um die paganen Kulte als unheilvoll und todbringend zu beschreiben. ${ }^{18}$ Dazu tritt die Vorstellung, dass der ,Aberglaube، wie ein Gift (venenum; mortiferum virus) und wie eine ansteckende Krankheit (funesta contagio) das römische Reich durchzieht und mit seinen rituellen Handlungen befleckt. ${ }^{19}$ Vor diesem Hintergrund verwendet Firmicus das Motiv des Blutes, um die unheilvolle und todbringende Wirkung der traditionellen Kulte noch weiter zu illustrieren. Gleichzeitig stellt er dem die positive Wirkung des Blutes Christi in diametraler Opposition gegenüber.

\section{Blut im Zusammenhang mit Gift und Heilung}

Nur kurz angesprochen wird von Firmicus das Gegensatzpaar von Gift und Heilung. In Kapitel 18 thematisiert er die Initiation in den Attiskult mit dem Vorwurf, dass dabei die christlichen Speisesakramente nachgeahmt würden. Diesen ,falschen' Sakramenten, die er als „verderbenbringenden Gifttrank“ (pestiferum veneni virus, err. 18,2) charakterisiert, stellt er die ,wahren', christlichen gegenüber, die den Menschen von genau diesem Gift befreien und ihm das Heil bringen können (err. 18,5.7-8). Über Zitate aus dem Johannesevangelium ${ }^{20}$ rückt Firmicus die zentrale Rolle Christi dabei in den Mittelpunkt und fügt wenig später noch hinzu: „Christi unsterbliches Blut haben wir getrunken, unserm Blut hat sich Christi Blut beigesellt. Dies ist das heilbringende Mittel gegen deine Verbrechen, das täglich aus dem versuchten Volke Gottes das todbringende Gift ausscheidet". ${ }^{21}$

\section{Blut als Nahrung der Dämonen}

Ein weiterer Aspekt, den Firmicus hinsichtlich der Verwendung des Blutes in den traditionellen Kulten anspricht, ist die Affinität der Dämonen zum Opferblut, ein in den christlichen Apologien häufig wiederkehrendes Thema. ${ }^{22}$

18 Firm. err. 16,3: busta sunt haec, ... non templa! 12,1: superstitionibus istius metuenda contagio; 16,4: Romanum orbem praesumptionis istius error funestus immaculet; 20,7: idolatriae ... funesta contagio.

19 Firm. err. 18,7; 21,3; 20,7 und 16,4.

20 Firmicus zitiert in err. 18,7 Joh 6,53 (Jesus als Brot des Lebens) und Joh 6,53(54): Nisi ederitis carnem fili hominis et biberitis sanguinem eius, non habebitis vitam in vobis. Der Bibeltext folgt Cyprian, Ad Quir. XXII.

21 Firm. err. 21,3: Christi immortalem sanguinem bibimus, nostro sanguini Christi sanguis adiunctus est. Hoc est salutare remedium scelerum tuorum, 〈quod> cottidie a vitiata dei plebe mortiferum virus exclusit. Angesprochen ist der Teufel.

22 So z.B. bei Tert. Apol. 22,6; Tert. scap. 2,8; Min. Fel. Oct. 27,2 und Orig. C. Cels. VIII,30. Dieselbe Position vertritt der neuplatonische Philosoph Porphyrios, De abst. II,42,2-3, weshalb Caseau eine Beeinflussung Firmicus' durch dieses Werk in Betracht zieht: „Firmicus a pu être très influencé par le De abstinentia 
Firmicus Maternus zufolge sammeln sich die bösen Dämonen in den Kultbildern der Tempel, da sie sich vom Blut der Opfertiere ernähren. ${ }^{23}$ Gleiches gilt für den Teufel, dessen Nachkommen die Dämonen sind, wobei es für sie unerheblich ist, ob das Opferblut von Tieren oder Menschen stammt. ${ }^{24}$ Die Gottheiten, in deren Kult diese Opfer dargebracht werden, sind Firmicus zufolge aber eine Erfindung des Teufels, der die Menschen davon zu überzeugen sucht, dass die Verehrung dieser vorgeblichen Götter zu ihrem Nutzen wäre. In Wirklichkeit aber betrüge der Teufel die Menschen, denn tatsächlich profitieren nur er und die Dämonen von den Opfern, während die Menschen durch diese Kulthandlungen ins Verderben gestürzt werden. ${ }^{25}$ Die Opfer bewirkten zudem, dass sich der Mensch zu den falschen Wesenheiten in Beziehung setzt und vom wahren Gott entfernt, wodurch er schlimmste Strafen auf sich ziehen kann. ${ }^{26}$

In der Vorstellung der Nichtchristen dienen die Opfer dazu, die Beziehung zur Gottheit aufrechtzuerhalten bzw. wiederherzustellen. Firmicus hingegen zeigt, dass diese Opfer das aber gerade nicht tun, sondern die Beziehung des Menschen zum wahren Gott zerstören und ihn stattdessen auf die Seite des Feindes, d.h. des Teufels, stellen. ${ }^{27}$ Seiner Ansicht nach besteht die Aufgabe des Christen darin, die Dämonen mithilfe der ihm durch die Gnade Jesu Christi zuteil gewordenen Macht auszutreiben, wo immer er sie findet. ${ }^{28}$

\section{Blut im Zusammenhang mit Reinheit und Befleckung}

Im Rahmen kultischer Handlungen wurde der Dichotomie von Reinheit und Befleckung besondere Aufmerksamkeit geschenkt, da kultische Reinheit die Voraussetzung jeglicher

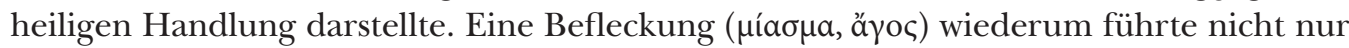
dazu, dass der Betroffene rituell unrein wurde und somit kultische Handlungen nicht mehr korrekt vollziehen konnte, sondern wurde auch als gefährlicher und ansteckender

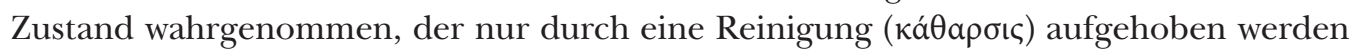
konnte. ${ }^{29}$ In diesem Zusammenhang tritt der ambivalente Status des Blutes besonders

dont il reprend les éléments critiques à l'égard des sacrifices“ (Caseau 2007: p. 52). Neben dem in 13,4 erwähnten Werk Über die Philosophie aus Orakeln kannte Firmicus sicher die Vita Plotini (math. I,7,14-22 mit Henry 1934: pp. 25-43). Die Kenntnis weiterer Werke des Porphyrios ist wahrscheinlich, lässt sich aber nicht sicher belegen. Da auch Firmicus' christliche Quellen umstritten sind, muss offen bleiben, woher die Vorstellung der opferblutaffinen Dämonen stammt.

23 Firm. err. 13,4: Sed in ipso simulacro sicut in ceteris ex assiduis sacrificiis immundi daemonum spiritus colliguntur. nihil enim operantur victimae et cruor ex assidua pecorum caede profusus, nisi et daemonum substantia, qui diaboli procreatione generantur, ex isto sanguine nutriatur.

Firm. err. 26,2. Nach err. 13,4 sind die Dämonen diaboli procreatione generantur.

25 Firm. err. 26,2.4.

26 Die Konsequenz dessen droht Firmicus in err. 28,10 mit einem Zitat aus Ex 22,19 an: Sacrificans diis eradicabitur praeter domino solo.

27 In err. 21,1 bezeichnet Firmicus den Teufel als nequissimum hostem generis humani.

28 Firm. err. 28,10 und 13,6.

29 Hoessly (2001: p. 44). Zu den antiken Reinheitsvorschriften vgl. Wächter (1910). Für das Thema Befleck- 
deutlich hervor. Einerseits wurde dem Blut reinigende Kraft zugeschrieben, andererseits konnte gerade die Befleckung mit Blut Ursprung gravierender Unreinheit sein. ${ }^{30}$ Lennon hat auf die Neigung der Apologeten von Tertullian an, die an das Christentum herangetragenen Vorwürfe umzukehren und auf die Ankläger zurückzuwenden, hingewiesen und vorgeschlagen, diese Verkehrung der Verhältnisse auch auf die Vorstellungen von Reinheit und Unreinheit zu beziehen. ${ }^{31}$ Firmicus Maternus liefert für diese Umkehrung ein weiteres Beispiel.

Das Motiv der Befleckung zieht sich in De errore durch die gesamte Darstellung der traditionellen Kulte, die immer wieder als besudelte Wahnvorstellungen und unheilvolle Irrtümer dargestellt werden, die den römischen Erdkreis beflecken. ${ }^{32}$ Die wichtigste Quelle der Befleckung ist bei Firmicus dabei das Blut, das in den Riten der traditionellen Kulte verwendet wird und mit dem sich der Teufel wie auch die Dämonen besudelt haben. ${ }^{33}$ Aus kultischen Handlungen, die im Zusammenhang mit Blut und diesen Wesen stehen, ergibt sich somit eine Befleckung für die daran beteiligten Personen. Ohne Reinigung ist diesen fortan ein normaler Kontakt mit der Gottheit nicht mehr möglich. ${ }^{34}$

Diesen Zusammenhang verdeutlicht Firmicus insbesondere in Kapitel 27. Die Passage gehört in den Kontext der ,teuflischen Nachahmung، der christlichen Sakramente, mit denen er sich über den zweiten Hauptteil hinweg beschäftigt. In 27,4 beschreibt Firmicus einen Ritus, bei dem der Teufel seine Anhänger bei Nacht einen Baum weihen und an dessen Fuß einen Widder opfern lässt. Firmicus kritisiert, dass es sich dabei um eine Nachahmung von Abrahams Opfer und des Passahs handle, obwohl diese Ereignisse lediglich die Vorausverkündung des wahren Passahs, d.h. des Todes Jesu Christi gewesen seien, der als Lamm Gottes bezeichnet werde. ${ }^{35}$ Das Kapitel schließt mit folgender Passage:

„Für das Heil der Menschen wird das verehrungswürdige Blut dieses Lammes vergossen, auf daß der Sohn Gottes seine Heiligen durch die Vergießung seines kostbaren Blutes erkaufe, damit diejenigen, die durch Christi Blut erlöst werden, zuvor durch die Majestät des unsterblichen Blutes geweiht werden. Niemanden hat das vor den Götzenbildern vergossene Blut

ung ist nach wie vor Parkers Studie grundlegend. Er zählt neben Blutvergießen u. A. auch Sakrilegien zu

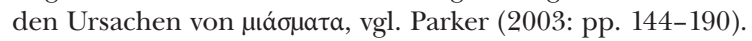

30 Requena Jiménez (2009: p. 67). Eines der berühmtesten Beispiele für die reinigende Wirkung des Blutes dürfte die Reinigung des Orest sein (vgl. z.B. Aesch. Eum. 283 sowie die Untersuchung dieses Falls bei Hoessly 2001: pp. 99-149). Neben anderen Beispielen verweist Requena Jiménez auf Suet. Galba 18,1, wo die Befleckung Galbas mit dem Blut eines Opferstiers als Todesomen gedeutet wird.

31 Lennon (2010: p. 383).

32 So z.B. in err. 16,4: Romanum orbem praesumptionis istius error funestus immaculet und 20,1: conmaculatas superstitiones.

33 Firm. err. 26,2: humanarum te etiam victimarum frequenter sanguine cruentasti; die Dämonen sind immundi ... spiritus (err. 13,4) und contaminatos ac pollutes spiritus (err. 28,10).

34 Hoessly (2001: pp. 44, 51).

35 Firm. err. 27,4-5.7. Für die Bezeichnung Jesu als Lamm Gottes beruft sich Firmicus in err. 27,5-8 auf Jes 53,7f., Jer 11,18f., Off 5,6-10 und Joh 1,29. 
erlöst, und damit das Blut von Tieren elende Menschen nicht täusche oder verderbe, ... Dieses Blut befleckt, erlöst nicht und vernichtet auf mancherlei Wegen den Menschen im Tode. Unglückselige sind es, die durch die Vergießung des gottlosen Blutes besudelt werden. Dieses Taurobolium oder Kriobolium übergießt dich mit dem Schmutz des verbrecherischen Blutes." 36

Die befleckende Wirkung des Blutes zeigt sich für Firmicus nicht nur im Opferblut der traditionellen Kulte (aput idola profusus sanguis), sondern auch bei den Riten in den Mysterien, konkret bei den Taurobolien bzw. Kriobolien. Die Formulierung scelerata te sanguinis labe perfundit deutet darauf hin, dass Firmicus nicht nur auf eine spirituelle Befleckung, sondern tatsächlich auf eine physische Kontamination mit Blut anspielt. Verstärkt wird dieser Eindruck noch dadurch, dass er diese Befleckung in Kapitel 28,1 der Taufe gegenüberstellt:

„Möge darum dieser Schmutz abgewaschen werden, den du sammelst! Suche reine Quellen, suche saubere Wässer, damit dich dort nach vielen Befleckungen Christi Blut mit dem heiligen Geist weiß wäscht.“37

In diesen beiden Passagen tritt das Gegensatzpaar zwischen ,richtigem ' und ,falschem Blut sehr deutlich hervor: Das Blut, das in den nichtchristlichen Ritualen verwendet wird und reinigen soll, befleckt und führt zu ritueller Unreinheit. Im Gegensatz dazu bietet Christus als das Lamm Gottes das wahre Blut, das allein die Kraft hat, zu reinigen, frühere Befleckungen abzuwaschen und somit wieder für ein intaktes Verhältnis zwischen Menschen und Gottheit sorgt.

\section{Blut im Zusammenhang mit Erlösung und Verderben}

Die oben bereits zitierte Passage aus err. 27,8 zeigt neben der Dichotomie von Reinheit und Befleckung noch ein weiteres Gegensatzpaar, denn Firmicus stellt die Hoffnung auf Erlösung dem Verderben gegenüber. Weder das im Rahmen der traditionellen Opfer noch das in den Mysterien verwendete Blut vermag den Menschen zu erlösen, sondern führt ihn vielmehr ins Verderben (peremit in morte). Nur Christus hat die Macht, den

36 Firm. err. 27,8: Pro salute hominum agni istius venerandus sanguis effunditur, ut sanctos suos filius dei profusione pretiosi sanguinis redimat, ut qui Christi sanguine liberantur, maiestate prius inmortalis sanguinis consecrentur. Neminem aput idola profusus sanguis invenit, et ne cruor pecorum miseros homines aut decipat aut perdat, ... Polluit sanguis iste, non redemit, et per varios casus hominem peremit in morte. Miseri sunt qui profusione sacrilegi sanguinis cruentantur. Tauribolium istud vel criobolium scelerata te sanguinis labe perfundit. Ziegler, von dem die leicht angepasste Übersetzung stammt, geht von einer Lücke vor polluit aus, Turcan hingegen übersetzt durchgehend. Die Zugehörigkeit des Tauroboliums bzw. Krioboliums wie auch die Ausführung dieser Rituale wird in der Forschung kontrovers diskutiert. Für meine Darstellung genügt die Feststellung, dass bei diesen Ritualen Blut Verwendung fand, das von Firmicus mit der Hoffnung auf Erlösung verknüpft und dem Blut Christi gegenübergestellt wird.

37 Firm. err. 28,1: Laventur itaque sordes istae quas colligis. Quaere fontes ingenuos, quaere puros liquores, ut illic te post multas maculas cum spiritu sancto Christi sanguis incandidet. 
Menschen zu erlösen (redimere), weshalb sein Blut, „das verehrungswürdige Blut dieses Lammes“, vergossen wurde, um den Menschen so das Heil zu schenken. Firmicus betont die Erlöserrolle Jesu auch noch an anderer Stelle, so z.B. in err. 24,2-4, wo Christus als Überwinder des Todes dargestellt und als heilbringende Gottheit (salutare numen) bezeichnet wird. ${ }^{38}$

\section{Fazit}

Die Verwendung des Blutes in religiösen Ritualen wird von Firmicus mit Hilfe von Gegensatzpaaren beschrieben, wobei er die Assoziationen, die aus der Sicht der traditionellen Kulte an die Wirkung des Blutes geknüpft sind, unterläuft und ins Gegenteil verkehrt. Entgegen der dem Blut zugeschriebenen reinigenden Wirkung befleckt und verunreinigt es den Menschen. Es erlaubt ihm auch nicht, eine Beziehung zum wahren Gott herzustellen und ihn zu erlösen, sondern trennt von ihm und assoziiert ihn mit den Mächten des Bösen. Es wirkt wie schleichendes Gift und führt den Menschen letztendlich auf den Pfad des Verderbens.

Im Gegensatz dazu bietet nur Christus allein das ,wahre Blut', das die Macht hat, das Gift des Teufels zu entfernen, den Menschen von der Befleckung zu reinigen, ihn zu erlösen und ihm das ewige Heil zu schenken. Mit der Betonung der Rolle Christi als der Gottheit, deren Blut all diese Funktionen auf sich vereint und die allein den Menschen auf den Weg des Heils (via salutis, err. 28,9) führt, unterstreicht Firmicus das spezifische proprium der christlichen Lehre, gegen das die vielfältigen nichtchristlichen Rituale nicht bestehen können.

Gleichzeitig kann Firmicus mithilfe der negativen Zuschreibungen zu den traditionellen Kulten die Gefahr darstellen, die von deren Weiterexistenz - ganz gleich, ob sacra publica oder sacra privata - ausgeht: Jede weitere Tolerierung der Rituale, allen voran der Opfer, fördert nur den Teufel und seine Dämonen und bedeutet, dass das römische Reich fortwährend von der daraus resultierenden Unreinheit bedroht wird. Gebannt werden kann diese Gefahr aus Firmicus' Sicht letzten Endes nur durch die vollständige Vernichtung aller traditionellen Kulte.

Die scheinbare Ungenauigkeit hinsichtlich der Darstellung der Mysterien entpuppt sich bei genauerem Hinsehen somit als Strategie des Autors, auf die Francesco Massa bereits hingewiesen hat: „Potrebbe trattarsi di una conseguenza della volontà di ,spersonalizzare“ i culti misterici, perché ciò che interessa a Firmico è una condanna della religio profana nella sua interezza. È quasi come se Firmico Materno procedesse alla costruzione di una sorta di ,fenomenologia‘ dei culti misterici di origine orientale, al di là delle loro specifiche identità“. ${ }^{9}$

38 Cf. auch err. 18,7f., wo Firmicus neben der heilsamen Wirkung des Blutes Christi auch das dadurch erlangte ewige Leben betont, das im Gegensatz zu den nichtchristlichen Ritualen steht, die den Tod bringen.

Massa (2013: p. 507). 
Die einzelnen Mysterien in ihren Details sind für Firmicus nicht von entscheidender Bedeutung, da sie letzten Endes allesamt auf denselben Ursprung, auf die Lehre des Teufels (diaboli disciplina, err. 18,1) zurückgeführt werden können. Insgesamt zielt seine Darstellung auf die Konstruktion zweier scheinbar homogener Systeme ab, d.h. der christlichen Lehre, vom höchsten Gott eingesetzt und durch die Wahrheit der Schrift belegt, und ihrer teuflischen Nachahmung, die zueinander in diametraler Opposition stehen. ${ }^{40}$ Die Verwendung des Motivs Blut untermauert diese Opposition zusätzlich: Während Reinheit, Gottesnähe und Erlösungshoffnung auf der einen Seite stehen, kann Firmicus mit Hilfe der Zuschreibung von Unreinheit, todbringendem Verderben und der unheilvollen Präsenz böser Mächte die vielfältigen Facetten der ,heidnischen Irrtümer' verdeutlichen.

Im Hinblick auf Vorbehalte gegen die kultische Verwendung von Blut steht Firmicus' Schrift um die Mitte des vierten Jahrhunderts nicht allein, sondern fügt sich in einen zeithistorischen und geistesgeschichtlichen Kontext ein, in dem die blutigen Opfer in mehrerlei Hinsicht thematisiert wurden. Das Verbot der Opfer unter Constans 341 zeigt die grundsätzliche Bereitschaft der Kaiser zu dieser Zeit mit Gesetzen gegen die traditionellen Kulte vorzugehen. ${ }^{41}$ Firmicus dürfte sich über diese Haltung im Klaren gewesen sein und gewusst haben, dass er mit seinen Forderungen in den Kreisen Gleichgesinnter auf Zustimmung stoßen würde. ${ }^{42}$ Abseits der Gesetzgebung wurde die Zulässigkeit des blutigen Opfers auch in neuplatonischen Kreisen diskutiert. Während z.B. Porphyrios diese Kultpraxis als für einen Philosophen unangemessen zurückwies, verteidigte Iamblichos sie als berechtigt. ${ }^{43}$ Auch diese Diskussion scheint Firmicus vertraut gewesen zu sein, denn schon in der Mathesis hatte er eine gewisse Skepsis gegenüber blutigen Opfern geäußert und unter Verweis auf Pythagoras und Porphyrios einem spirituellen Kult den Vorzug gegeben. ${ }^{44}$ Seine Beschreibung des summus deus und die zentrale Rolle, die er der Reinheit und dem Weg zum Heil einräumt, legen die Schlussfolgerung nahe, dass Firmicus vor seiner Konversion dem Neuplatonismus nahe gestanden hat. ${ }^{45}$ Die Schrift De errore profanarum religionum zeigt, dass zahlreiche Elemente dieser philosophischen

40 Massa (2013: pp. 499, 507) und im Anschluss daran Gassman (2020: p. 60).

41 CTh XVI,10,2. Das Gesetz von 341 zieht kein systematisches Vorgehen gegen die Nichtchristen nach sich; ein Gesetz aus dem Folgejahr, CTh XVI,10,3, schützt sogar ausdrücklich Tempel außerhalb der Mauern Roms. Die Datierung von CTh XVI,10,3 folgt Seeck (1984: p. 191).

42 Ahmed (2017: p. 184). Inwiefern sprachliche Parallelen zwischen einzelnen Gesetzen und Passagen aus De errore auf Bezüge zwischen beiden Texten hindeuten, ist umstritten. So wurde beispielsweise ein Zusammenhang zwischen CTh XVI,10,3 (penitus eruenda) und err. 16,4 (penitus ... delenda) sowie zwischen CTh XVI,10,4 (gladius ultor) und err. 29,2 (gladius vindex) in Betracht gezogen; die Datierung des zweiten Gesetzes ist jedoch umstritten. Woudhuysen macht darauf aufmerksam, dass gladius ultor auch in anderen Gesetzen des Constans zu finden ist, darunter in CTh IX,7,3 und II,1,1. Er deutet die Parallelen als Zeichen der Unterstützung der kaiserlichen Politik (Woudhuysen 2018: p. 176).

43 Porph. De abst. II,42,2-43,1. Iamblichos diskutiert in De myst. V die von Porphyrios in Ad Aneb. vorgebrachten Einwände und rechtfertigt das Tieropfer u. A. in De myst. V,14 und VI,1.

44 Firm. math. II,30,10 und VII,1,1 = Fr. 488F Smith.

45 Cf. oben, Anm. 15 u. 16. Zum selben Ergebnis kommt Caseau (2007: p. 51). 
Strömung mit Firmicus' Ansichten des Christentums kompatibel waren, was ihn über seine Verwendung des Motivs Blut hinaus zu einem der zahlreichen bemerkenswerten Teilnehmer des kompetitiven, interreligiösen Diskurses um die Zulässigkeit und Bedeutung des blutigen Opfers machte. ${ }^{46}$

\section{Bibliographie}

\section{Quellen}

Bouffartigue, J., \& Patillon, M. (Eds.). (2018). Porphyre: De L'Abstinence, II: Livres II et III. Paris: Belles Lettres.

Clarke, E. C., Dillon, J. M., \& Hershbell, J. P. (2003). (Transl.). Iamblichus: On the Mysteries (Writings from the Graeco-Roman World, 4). Atlanta: Society of Biblical Literature.

Fiedrowicz, M., \& Barthold, C. (Transl.). (2012). Origenes: Contra Celsum. Gegen Celsus (Fontes Christiani, 50, 4). Freiburg i. Br.: Herder.

Forbes, C. A. (Transl.). (1970). The Error of the Pagan Religions (Ancient Christian Writers, 37). Turnhout: Brepols.

Georges, T. (Transl.). (2015). Tertullian: Apologeticum. Verteidigung des christlichen Glaubens (Fontes Christiani, 62). Freiburg i. Br.: Herder.

Kroll, W., Skutsch, F., \& Ziegler, K. (Eds.). (1968). Iulii Firmici Materni: Matheseos Libri VIII. Libros IV posteriores cum praefatione et indicibus continens. Stuttgart - Leipzig: Teubner.

Kroll, W., \& Skutsch, F. (Eds.). (1968). Iulii Firmici Materni: Matheseos Libri VIII. Fasciculus Prior: Libros IV Priores Continens. Stuttgart - Leipzig: Teubner.

Kytzler, B. (Ed.). (1992). M. Minuci Felicis Octavius. Stuttgart - Leipzig: Teubner.

Martinet, H. (Transl.). (2014). C. Suetonius Tranquillus: Die Kaiserviten. De vita caesarum. Berühmte Männer. De viris illustribus. Lateinisch-deutsch. Berlin: Akademie-Verlag.

Monat, P. (Ed.). (1994). Firmicus Maternus: Mathesis, II: Livres III-V. Paris: Belles Lettres.

Monat, P. (Ed.). (2000). Lactance: Institutions Divines. Livre V (Vol. I ; Sources Chrétiennes, 204). Paris: Cerf.

Monat, P. (Ed.). (2002). Firmicus Maternus: Mathesis, I: Livres I et II. Paris: Belles Lettres.

Monat, P. (Ed.). (2003). Firmicus Maternus: Mathesis, III: Livres VI-VIII. Paris: Belles Lettres.

Moreschini, C., \& Podolak, P. (Eds.). (2006). Tertulliano: Opere apologetiche. Roma: Città Nuova.

Pastorino, A. (Ed.). (1969). Iuli Firmici Materni De errore profanarum religionum. Firenze: La Nuova Italia.

Schleyer, D. (Transl.). (2002). Tertullian: De praescriptione haereticorum. Vom prinzipiellen Einspruch gegen die Häretiker (Fontes Christiani, 42). Turnhout: Brepols.

Smith, A. (Ed.). (1993). Porphyrii Philosophi Fragmenta. Stuttgart - Leipzig: Teubner.

Smyth, H. W. (Ed.). (1926). Aeschylos, 2: Eumenides. Cambridge, MA: Harvard University Press.

Turcan, R. (Ed. \& Transl.). (2002). Firmicus Maternus: L'erreur des religions païennes. Paris: Belles Lettres.

46 Die Diskussion um die Tieropfer der traditionellen Kulte über die longue durée hinweg bildet den Gegenstand meines Dissertationsvorhabens. 
Ulrich, J. (Ed. \& Transl.). (2019). Justin: Apologien (Kommentar zu frühchristlichen Apologeten, 4, 5). Freiburg i. Br.: Herder.

Weber, R. (Ed.). (1972). Sancti Cypriani Episcopi Opera. Ad Quirinum (Corpus Christianorum, Series Latina III, I). Turnhout: Brepols.

Ziegler, K. (Ed.). (1953a). Iuli Firmici Materni V. C.: De errore profanarum religionum. München: Hueber.

Ziegler, K. (Ed.). (1953b). Julius Firmicus Maternus, Senator: Vom Irrtum der heidnischen Religionen (Das Wort der Antike, 3). München: Hueber.

\section{Sekundärliteratur}

Ahmed, L. (2017). Bilder von den Anderen. Christliches Sprechen über Heiden bei den lateinischen Apologeten (Jahrbuch für Antike und Christentum, Ergänzungsband, Kleine Reihe, 14). Münster: Aschendorff.

Caseau, B. (2007). Firmicus Maternus. Un astrologue converti au christianisme ou la rhétorique du rejet sans appel. In D. Tollet (Ed.), La religion que j'ai quittée. Religions dans l'histoire (pp. 39-63). Paris: PUPS.

Festugière, A.-J. (1998). Trois dévots païens. Paris: Arfuyen.

Gaddis, M. (2005). There Is No Crime for Those Who Have Christ. Religious Violence in the Christian Roman Empire. Berkeley: University of California Press.

Gassman, M. P. (2020). Worshippers of the Gods. Debating Paganism in the Fourth-Century Roman West. Oxford: Oxford University Press.

Gersh, St. (2018). §139. Firmicus Maternus. In Ch. Riedweg et al. (Eds.), Die Philosophie der Antike, 5/2: Philosophie der Kaiserzeit und der Spätantike (pp. 1634-1641). Basel: Schwabe.

Henry, P. (1934). Plotin et l'Occident. Firmicus Maternus, Marius Victorinus, Saint Augustin et Macrobe (Spicilegium Sacrum Lovaniense, 15). Louvain: Spicilegium Sacrum Lovaniense.

Hoessly, F. (2001). Katharsis: Reinigung als Heilverfahren. Studien zum Ritual der archaischen und klassischen Zeit sowie zum Corpus Hippocraticum (Hypomnemata, 135). Göttingen: Vandenhoeck \& Ruprecht.

Hübner, W., \& Wlosok, A. (1989). Firmicus Maternus (Iulius Firmicus Maternus iunior). In R. Herzog (Ed.), Restauration und Erneuerung. Die lateinische Literatur von 284 bis 374 n. Chr. (pp. 84-93). München: Beck.

Jones, A. H. M., Martindale, J. R., \& Morris, J. (1987). The Prosopography of the Later Roman Empire 1: A.D. 260-395. Cambridge: Cambridge University Press.

Massa, F. (2013). Confrontare per distruggere. Firmico Materno e l'origine diabolica dei culti orientali. Studi e Materiali di Storia delle Religioni, 79(2), 493-509.

Parker, R. (2003). Miasma. Pollution and Purification in Early Greek Religion. Oxford: Clarendon Press.

Requena Jiménez, M. (2009). Kleidung, Blut und Befleckung in der paganen Welt der Antike. In S. Schrenk et al. (Eds.), Kleidung und Identität in religiösen Kontexten der römischen Kaiserzeit. Altertumswissenschaftliches Kolloquium in Verbindung mit der Arbeitsgruppe „Kleidung und Religion“, Projekt DressID, Rheinische Friedrich-Wilhelms-Universität Bonn, 30. und 31. Oktober 2009 (Mannheimer Geschichtsblätter Sonderveröffentlichung, 4; pp. 65-70). Regensburg: Schnell \& Steiner. 
Seeck, O. (1984). Regesten der Kaiser und Päpste für die Jahre 311 bis 476 n. Chr. Frankfurt a. M.: Minerva.

Wächter, T. (1910). Reinheitsvorschriften im griechischen Kult (Religionsgeschichtliche Versuche und Vorarbeiten, IX/1). Gießen: Töpelmann.

Woudhuysen, G. (2018). Uncovering Constans' Image. In D. W. P. Burgersdijk, \& A. Ross (Eds.), Imagining Emperors in the Later Roman Empire (Cultural Interactions in the Mediterranean, 1; pp. 158-182). Leiden: Brill.

Bettina Reese / bettina.reese@altertum.uni-halle.de

Institute of Classical Antiquity

Martin Luther University of Halle-Wittenberg, Faculty of Philosophy I

Universitätsplatz 12, 06108 Halle (Saale), Germany 
